\title{
Bose-Einstein correlations in various collision systems and energies measured with the CMS experiment
}

\author{
Sandra S. Padula (on behalf of the CMS Collaboration) ${ }^{* \dagger}$ \\ UNESP - IFT \\ E-mail: sandra.padula@cern.ch
}

\begin{abstract}
Two-particle, quantum-statistical (Bose-Einstein) correlations are measured with the CMS experiment in pp collisions at $0.9,2.76$ and $7 \mathrm{TeV}$ center-of-mass energies, as well as in $\mathrm{pPb}$ and peripheral $\mathrm{PbPb}$ collisions, respectively at 5.02 and $2.76 \mathrm{TeV}$ center-of-mass energy per nucleon. The analysis is performed as a function of both the charged particle multiplicity and the average transverse pair momentum. The measurement is performed with charged particles and the full available statistics in pp collisions, being also compared to previous one-dimensional results from CMS. The analysis is then extended to two- and three-dimensions for investigating the source lengths of homogeneity in different spatial directions. Furthermore, low $p_{T}$ pions and kaons, which are identified from their energy loss in the silicon tracker, are used to further extend the measurements to $\mathrm{pp}, \mathrm{pPb}$ and $\mathrm{PbPb}$ systems at the available energies. Radius fit parameters on the order of $1-5 \mathrm{fm}$ are found, the largest values corresponding to very high multiplicity $\mathrm{pPb}$ interactions and for similar multiplicity $\mathrm{PbPb}$ collisions.
\end{abstract}

XXIII International Workshop on Deep-Inelastic Scattering,

27 April - May 12015

Dallas, Texas

\footnotetext{
* Speaker.

${ }^{\dagger}$ Partially supported by the São Paulo Research Foundation (FAPESP) under Grant N. 2013/01907-0 and by the Conselho Nacional de Desenvolvimento Científico e Tecnológico (CNPq) under Grant N. 307249/2012-3.
} 
Two-particle Bose-Einstein correlations (BEC) play a crucial rôle for revealing the size and shape of the source emitting region in high-energy collisions. One-dimensional (1-D) BEC were investigated in the Compact Muon Solenoid (CMS) experiment in terms of the invariant pair relative momentum $q_{\text {inv }}=\sqrt{-q^{\mu} q_{\mu}}=\sqrt{-\left(k_{1}-k_{2}\right)^{2}}$ ( $k_{i}$ is the momentum of each particle in the pair) and reported in Refs. [1, 2]. This work [3, 4] extends those measurements to multidimensions by investigating BEC in terms of various components of $\mathbf{q}$, exploring the source extent in different directions. Two techniques are adopted in this analysis: the first is the same as in [1,2], here called double ratio method, applied to charged hadrons produced in pp collisions at 2.76 and $7 \mathrm{TeV}$ [3]; the second is a new data-driven method [4], which extends the analysis further to $\mathrm{pp}, \mathrm{pPb}$ and $\mathrm{PbPb}$ collisions at various energies for identified pions and kaons. The BEC is also studied as a function of the average transverse pair momentum $\left(\mathbf{k}_{T}=\left(\mathbf{k}_{T_{1}}+\mathbf{k}_{T_{2}}\right) / 2\right)$ and of the charged particle multiplicity of the event ( $N_{\mathrm{ch}}$ in the charged hadron case, and $N_{\text {tracks }}$ for identified pions and kaons).

A complete description of the CMS detector can be found in Ref. [5]. The tracker is the most important part of the detector for this analysis and measures charged particles within the $|\eta|<2.4$ range. The pseudorapidity $\eta$ of a particle is defined in the LHC laboratory frame as $\eta=-\ln [\tan (\theta / 2)]$, where $\theta$ is the polar angle with respect to the beam direction.

The part of this analysis using the double ratio technique adopts the same event and track selections as in Refs. [1,2] and considers minimum bias (MB) events from pp collisions at $\sqrt{s}=2.76$ (3.4 million events in data and 2 million from Pythia-6 [6]) and $7 \mathrm{TeV}$ (total of 43 million events in data 33 million from Pythia-6 [6]), as described in detail in Ref. [3]. The part using the datadriven technique considers MB pp collisions at $\sqrt{s}=0.9,2.76$ and $7 \mathrm{TeV}$ (respectively, 8.97, 9.62, and 6.20 million events), $\mathrm{pPb}$ collisions at $\sqrt{s_{N N}}=5.02 \mathrm{TeV}$ (8.95 million) and $\mathrm{PbPb}$ collisions at $\sqrt{s_{N N}}=2.76 \mathrm{TeV}$ (3.07 million $60-100 \%$ peripheral events, where $0 \%$ means central head-on collision and $100 \%$ corresponds to fully peripheral); complete information about the corresponding event and track selections can be found in Ref. [4], as well as the procedure for identifying pions and kaons through their energy loss in the silicon tracker with very high (>95\%) purity.

The signal containing the BEC using the technique in Refs. [1,2] is formed by pairing all equal charged tracks from the same event originating from the primary vertex, with $|\eta|<2.4$ and $p_{T}>0.2 \mathrm{GeV}$. A histogram is filled with such pair distribution in bins of $N_{c h}$, of $k_{T}$, as well as of the relative momentum of the pair, i.e., either in terms of $q_{\text {inv }}$ in the one-dimension (1-D), or in terms of its components $\left(q_{T}, q_{L}\right)$ in two dimensions (2-D), or $\left(q_{O}, q_{S}, q_{L}\right)$ in three-dimensions (3D). The components $q_{L}$ (longitudinal) and $q_{T}$ (transverse) are, respectively, the relative momentum along and transverse to the beam direction. In the 3-D case, $\mathbf{q}_{T}$ is projected further in orthogonal directions $\mathbf{q}_{O}$ (outwards) (along $\mathbf{k}_{T}$ ), and $\mathbf{q}_{S}$ (sidewards), orthogonal to both $\mathbf{q}_{O}$ and $\mathbf{q}_{L}$.

The background or reference sample can be formed by pairing particles (all charge combinations) from mixed events having $\eta$ within $-2.4 \leq \eta \leq-0.8,-0.8 \leq \eta \leq 0.8$ or $0.8 \leq \eta \leq 2.4$ [2].

A single ratio $(S R)$ is then defined, with the signal pair distribution as numerator and the reference sample as denominator with the appropriate normalization, i.e.,

$$
\operatorname{SR}\left(q_{\mathrm{inv}}\right)=\left(\frac{N_{\mathrm{ref}}}{N_{\mathrm{sig}}}\right) \frac{\left(\mathrm{d} N_{\mathrm{sig}} / \mathrm{d} q_{\mathrm{inv}}\right)}{\left(\mathrm{d} N_{\mathrm{ref}} / \mathrm{d} q_{\mathrm{inv}}\right)},
$$

where $N_{\text {sig }}$ is obtained by summing up the pair distribution for all the events in the signal and, similarly, $N_{\text {ref }}$ in the reference sample. 

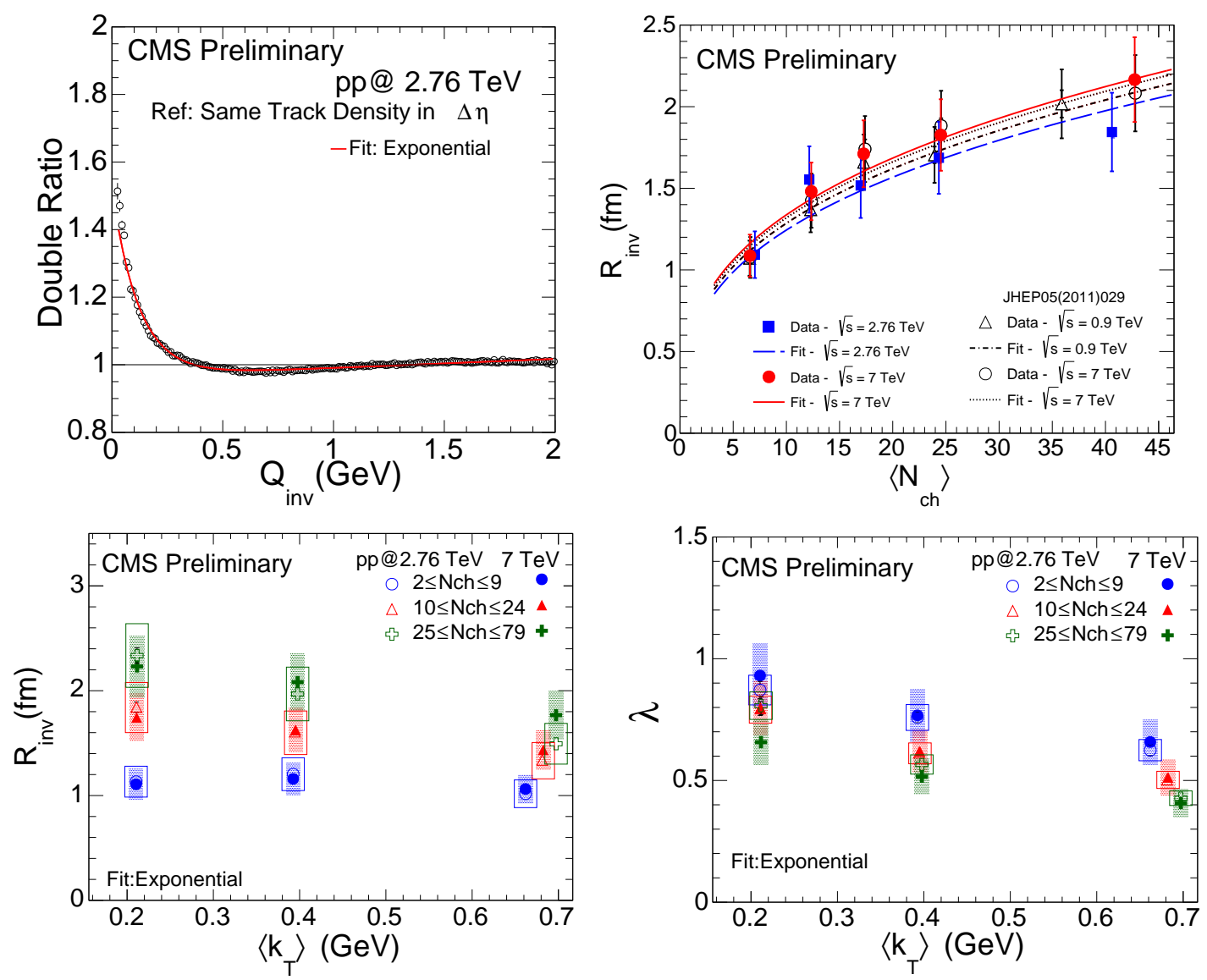

Figure 1: Results obtained with the double ratio technique and with the exponential fit function. The top panel shows 1-D BEC function versus $q_{\text {inv }}$ for pp collisions at $2.76 \mathrm{TeV}$ (left) and the fit parameter $R_{\text {inv }}$ as a function of $\left\langle N_{\mathrm{ch}}\right\rangle$ (acceptance and efficiency corrected), for pp collisions at $2.76 \mathrm{TeV}$ and $7 \mathrm{TeV}$ (right); results from Ref. [2] at 0.9 and $7 \mathrm{TeV}$ are also included in the plot (inner error bars represent the statistical uncertainties, while outer error bars the statistical and systematic uncertainties added in quadrature). The bottom panel shows $R_{\text {inv }}$ and $\lambda$ in three bins of $<N_{\mathrm{ch}}>$ and $k_{T}$.

For obtaining the BEC parameters in this method $[1,2]$ a double ratio (DR) is then formed as $\operatorname{DR}\left(q_{\text {inv }}\right)=S R\left(q_{\text {inv }}\right) / S R\left(q_{\text {inv }}\right)_{\mathrm{MC}}$, where $S R\left(q_{\text {inv }}\right)_{\mathrm{MC}}$ is the single ratio as in Eq. (1), computed in the same way as in data but with simulated events generated without BEC. The measurement is performed in two frames: the center-of-mass (CM) and in the Local Co-Moving System (LCMS), where $k_{L}=\left(k_{L_{1}}+k_{L_{2}}\right) / 2$, the longitudinal component of the pair average momentum, vanishes.

The double-ratio procedure was initially adopted in Refs. $[1,2]$ to reduce the bias due to the construction of the reference samples. Besides, it has also the advantage of reducing the sources of bias due to track inefficiency and other detector-related effects, as well as removing non-BoseEinstein correlations that may remain in the single ratios due to the mixing procedure, allowing for the BEC to emerge without such distortions.

The femtoscopic correlations in discussion reflect not only the quantum statistics of the pair of identical particles, but is also sensitive to the underlying dynamics. In the case of charged hadrons 

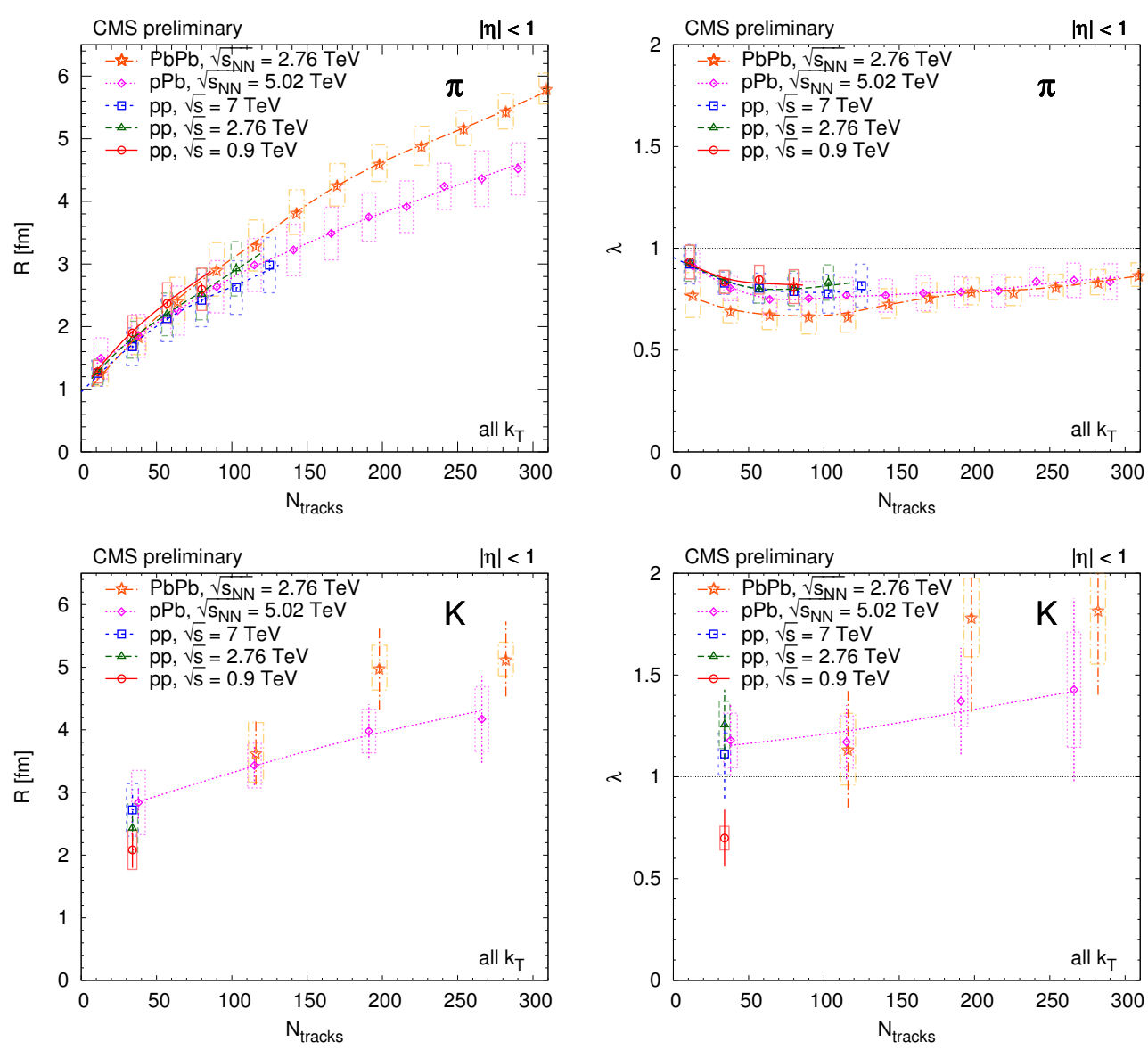

Figure 2: Results for the data-driven technique and exponential fit function: 1-D parameters $R$ and $\lambda$ versus charged multiplicity $N_{\text {tracks }}$ (acceptance and efficiency corrected) for pions (top) and for kaons (bottom).

the Coulomb final state interaction unavoidably contaminate the signal sample. For pointlike sources it can be corrected by the Gamow factor which, in case of same charge and opposite charge pairs, is given by $G_{w}^{S S}\left(\eta_{w}\right)=\left|\Psi^{S S}(0)\right|^{2}=\frac{2 \pi \eta_{w}}{\exp \left(2 \pi \eta_{w}\right)-1}, \quad G_{w}^{O S}\left(\eta_{w}\right)=\left|\Psi^{O S}(0)\right|^{2}=\frac{2 \pi \eta_{w}}{1-\exp \left(-2 \pi \eta_{w}\right)}$, where $\eta_{w}=\alpha_{e m} m_{\pi} / q_{\text {inv }}, \alpha$ is the fine-structure constant, and $m$ is the particle mass. For Cauchy (Lorentz) type of source functions the Coulomb interactions are corrected by the full Coulomb factor $K\left(q_{\text {inv }}\right)=G_{w}\left(\eta_{w}\right)\left[1+\pi \eta_{w} q_{\text {inv }} R /\left(1.26+q_{\text {inv }} R\right)\right]$. In the case of MB pp collisions the correction provided by the Gamow factor coincides with the the full $K\left(q_{\text {inv }}\right)$ factor.

In the data-driven technique with particle identification, $\pi^{+} \pi^{+}, \pi^{-} \pi^{-}, \pi^{+} \pi^{-}, \mathrm{K}^{+} \mathrm{K}^{+}, \mathrm{K}^{-} \mathrm{K}^{-}$, $\mathrm{K}^{+} \mathrm{K}^{-}$, pair distributions are binned in the number of reconstructed charged tracks $N_{\text {tracks }}$ of the event, in the transverse pair momentum $k_{T}$, and also in the relative momentum variables, $q_{\mathrm{inv}}$, or in the LCMS of the pair, in terms of $\left(q_{T}, q_{L}\right)$ or $\left(q_{O}, q_{S}, q_{L}\right)$. The construction of the signal distribution is analogous to that described in Eq. (1) for the double ratio method. For the reference sample, another mixing procedure is adopted, in which particles from the same event are paired with particles from 25 preceding events. Only events belonging to the same multiplicity $\left(N_{\text {tracks }}\right)$ class are mixed. In this data-driven method, $S R\left(q_{\text {inv }}\right)$ as in Eq. (1) are considered for obtaining the 

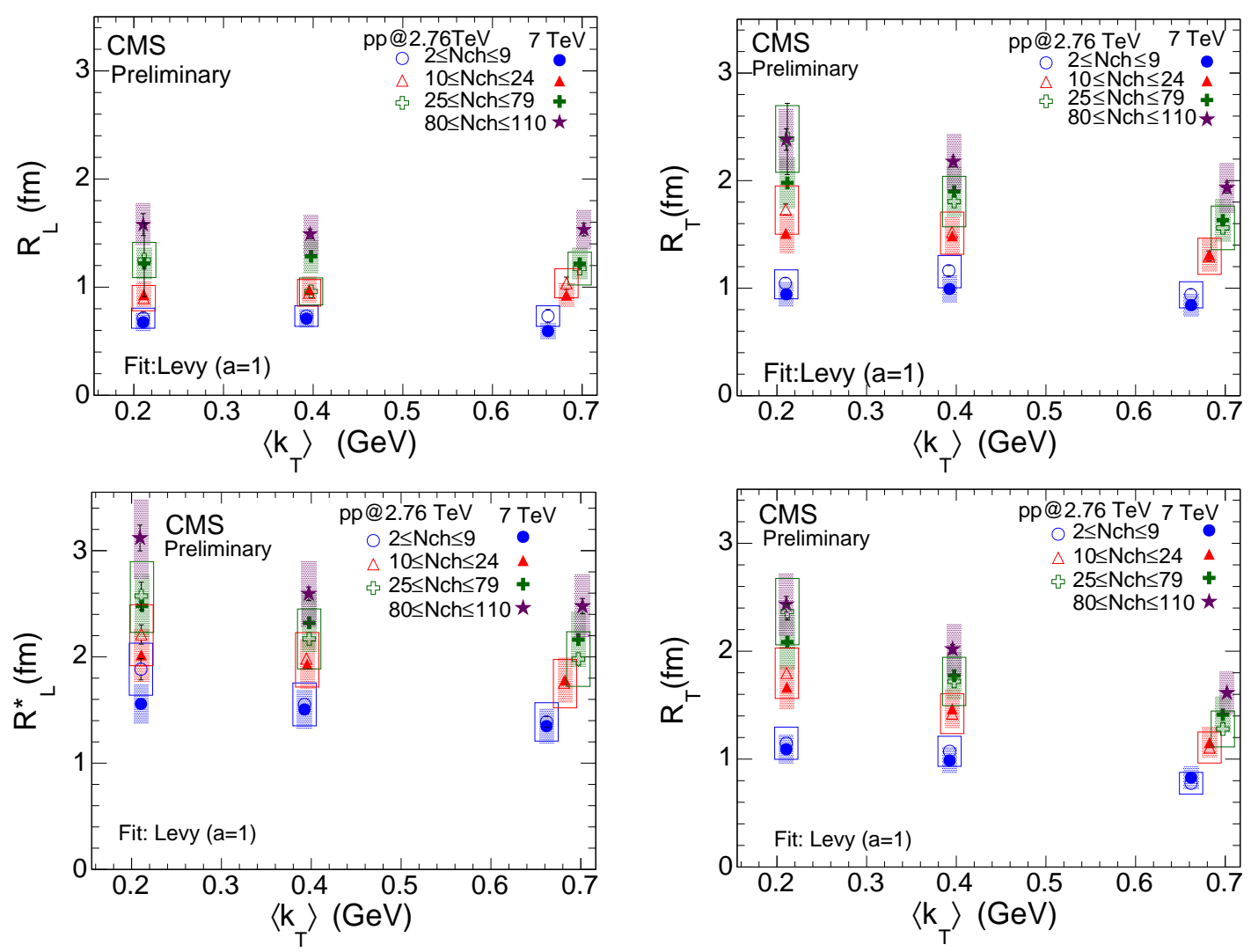

Figure 3: Results with the DR technique in 2-D: fit parameters $\left(R_{L}^{(*)}, R_{T}\right)$ obtained with the stretched exponential function are shown for pp collisions at 2.76 and $7 \mathrm{TeV}$ in the $\mathrm{CM}$ frame (top) and in the LCMS (bottom), as a function of $\left\langle k_{T}\right\rangle$ and for different $N_{\mathrm{ch}}$ bins. The statistical uncertainties are indicated by error bars, whereas the systematic ones are indicated by empty (at $2.76 \mathrm{TeV}$ ) or shaded boxes (at $7 \mathrm{TeV}$ ).

BEC fit parameters. However, these are usually contaminated by non-Bose-Einstein effects, even after correcting for Coulomb final state interactions. Such contaminations are mainly due to multibody decays of resonances and minijets (together called "cluster" contribution). Instead of using double ratios for eliminating such distorting effects, they are estimated with the aid of oppositesign particle correlations, and then a fraction of them is subtracted from the corresponding identical particle correlation functions, resulting in the filtered $C_{B E}\left(q_{\text {inv }}\right)$, equivalent to the $D R\left(q_{\text {inv }}\right)$ function in the double ratio technique. The details are found in Ref. [4].

The parameterizations used to fit the BEC functions in 1-D and 2-D (see Refs. [3] and [4] for the complete report, including the 3-D case) in terms of $q_{\text {inv }}$ and $\left(q_{L}, q_{T}\right)$ can be written as

$$
\begin{gathered}
D R\left(q_{\text {inv }}\right)=C\left[1+\lambda \exp \left\{-\left(q_{\text {inv }} R_{\text {inv }}\right)^{a}\right\}\right]\left(1+\delta q_{\text {inv }}\right) \sim C_{B E}\left(q_{\text {inv }}\right) \\
D R\left(q_{T}, q_{L}\right)=C\left\{1+\lambda \exp \left[-\left|q_{T}^{2} R_{T}^{2}+q_{L}^{2} R_{L}^{2}+2 q_{T} q_{L} R_{L T}^{2}\right|^{a / 2}\right]\right\} \times\left(1+\alpha q_{T}+\beta q_{L}\right) \sim C_{B E}\left(q_{T}, q_{L}\right) .
\end{gathered}
$$

For the class of Lévy stable distributions with index of stability $0<a \leq 2$ as in Eq. (3) the Bose-Einstein correlation function has a stretched exponential shape [7] ( $a=1$ corresponds to the 
exponential function and $a=2$, to the Gaussian distribution). In particular, for $a=1$, the exponential terms in Eq. (2) and (3) coincide with the Fourier transform of the source function $\rho(t, \vec{x})$, characterized by a Lorentzian or Cauchy distribution; the radius parameters $R_{\text {inv }}$ and $\left(R_{T}, R_{L}\right)$ correspond to the fitted lengths of homogeneity in 1-D and 2-D, respectively. In the above expressions, $\lambda$ is the intercept parameter (intensity of the correlation in the lowest bin of the pair relative momentum), $C$ is the overall normalization. The additional polynomial terms, proportional to the fit constants $\delta, \alpha, \beta$ describe possible long-range correlations. In Eq. (3) a frame-dependent crossterm proportional to $q_{T} q_{L}$ is present. However, when the analysis is performed in the LCMS, this cross-term does not contribute for sources symmetric along the longitudinal direction.
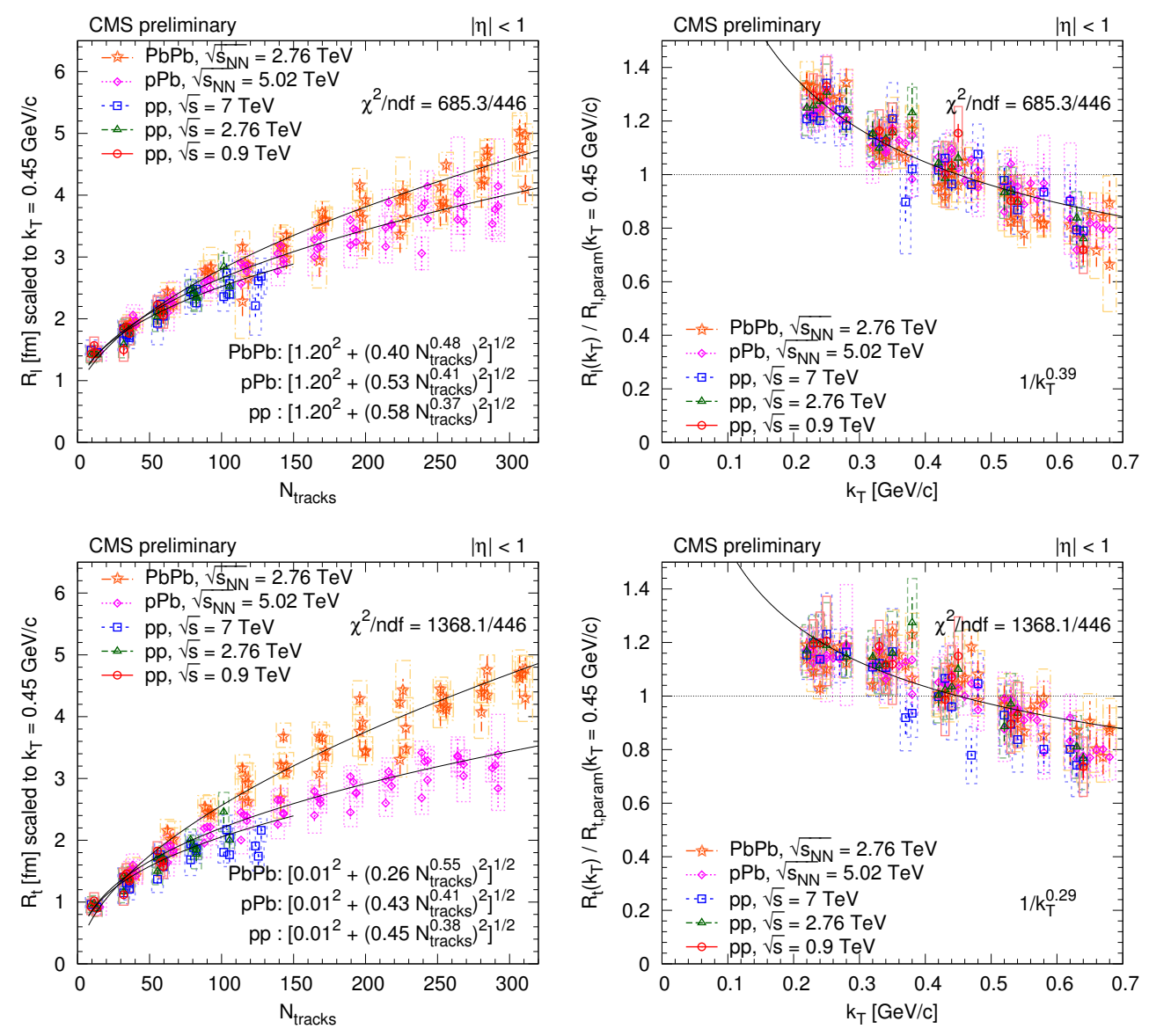

Figure 4: Left panel: radius fit parameters as a function of $N_{\text {tracks }}$ scaled to $k_{T}=0.45 \mathrm{GeV}$ with help of the parametrization $R_{\text {param }}$. Right panel: ratio of the radius fit parameter and the value of the parametrization $R_{\text {param }}$ at $k_{T}=0.45 \mathrm{GeV}$ as a function of $k_{T}$. The points were shifted to the left and to the right with respect to the center of the $k_{T}$ bin for better visualization $\left(R_{L}\right.$ is in the top panel and $R_{T}$, in the bottom panel).

Figure 1, exhibits results obtained with the double ratio technique and the exponential fit function. The top panel shows the 1-D correlation function (left) and radius fit parameter versus $N_{\mathrm{ch}}$ (right), where new results appear together with those from Ref. [2], suggesting a similar increase of $R_{\text {inv }}$ with $N_{\mathrm{ch}}^{1 / 3}$ and an approximate scaling with center-of-mass energy $\sqrt{s}$. The bottom panel shows $R_{\text {inv }}$ (left) and $\lambda$ (right) for pp collisions at 2.76 and $7 \mathrm{TeV}$, where $R_{\text {inv }}$ steadly increase with 
$N_{\text {ch }}$ and decrease with $k_{T}$ (at least for the largest charged multiplicity bins), a behavior which is expected for expanding systems. As for $\lambda$, it seems to decrease with $N_{\mathrm{ch}}$ and also slightly with $k_{T}$.

A similar trend can be seen in Figure 2 for results with the data-driven technique, showing 1-D invariant radius $R$ versus $N_{\text {tracks }}$ for all $k_{T}$, obtained with the exponential fit function for pions produced in $\mathrm{pp}, \mathrm{pPb}$ and peripheral $\mathrm{PbPb}$ collisions. Results correponding to events with similar number of tracks in the $\mathrm{pp}$ and $\mathrm{pPb}$ cases show compatible values of the radius fit parameters. In the case of kaons, the overall trend is the same, although the increase is less pronounced. As for $\lambda$, for all $k_{T}$, it seems to decrease with $N_{\text {tracks }}$ for pions and then saturate; for kaons an opposite trend is seen in the $\lambda$ behavior. The extracted lengths of homogeneity are in the range $1-5 \mathrm{fm}$, reaching the highest values for very high multiplicity $\mathrm{pPb}$, also for similar multiplicity $\mathrm{PbPb}$ collisions.

In Figure 3, 2-D results with the double ratio technique are shown in the CM frame (top) and in the LCMS (bottom). The behaviour is very similar in both frames, clearly showing that all fit parameters $R_{L}$ ( $R_{L}^{*}$, in the LCMS) and $R_{T}$ increase with increasing multiplicity $N_{\text {ch. An opposite }}$ behavior is oberved for $\lambda$. The lengths of homogeneity $R_{L}\left(R_{L}^{*}\right)$ and $R_{T}$ decrease with $k_{T}$ for larger multiplicities, similar to the observed in the 1-D case. Another interesting feature of the data that can be seen is that $R_{L}^{*}$ (LCMS) $>R_{L}(\mathrm{CM})$ for the same bins of $N_{c h}$ and $k_{T}$, an effect that could be related to the Lorentz boost contraction of the longitudinal length of homogeneity in the CM frame. Also noticeable is the fact that $R_{L}^{*}>R_{T}$ in the LCMS, suggesting a source elongated longitudinally. A similar trend is also observed in the 3-D case and with the data-driven method [3, 4].

In Figure 4, results with the data-driven technique show that, by fitting the radius parameters in differential bins of $N_{\text {tracks }}$ and $k_{T}$ with a product of two independent functions of these variables, i.e., $R_{\text {param }}\left(N_{\text {tracks }}, k_{T}\right)=\left[a^{2}+\left(b N_{\text {tracks }}^{\beta}\right)^{2}\right]^{1 / 2} \cdot\left(0.2 p_{T} G e V / k_{T}\right)^{\gamma}$, the dependences on multiplicity and pair momentum appear to factorize. In some cases the fit radii are less sensitive to the type of colliding system and center-of-mass energy. The minimal radius $a$ and the exponents $\gamma$ of $k_{T}$ are kept the same for a given radius component, for all collision types. This minimal radius $a$ can be connected to the size of the proton, while the power-law dependence on $N_{\text {tracks }}$ is often attributed to the freeze-out density of hadrons. The ratio of radius parameter and the value of the above parametrization at $k_{T}=0.45 \mathrm{GeV}$ as a function of $k_{T}$ is shown in the right column of Figure 4.

\section{References}

[1] CMS Collaboration, Phys. Rev. Lett. 105, 32001 (2010).

[2] CMS Collaboration, JHEP05, 029 (2011).

[3] CMS Collaboration, Multidimensional analysis of Bose-Einstein correlations in pp collisions at 2.76 and $7 \mathrm{TeV}$, CMS-PAS-FSQ-13-002 (2014); https://twiki.cern.ch/twiki/bin/view/CMSPublic/PhysicsResultsFSQ13002.

[4] CMS Collaboration, Femtoscopy with identified charged hadrons in $p p, p P b$ and peripheral $\mathrm{PbPb}$ collisions at LHC energies, CMS-PAS-HIN-14-013 (2014);

https://twiki.cern.ch/twiki/bin/view/CMSPublic/PhysicsResultsHIN14013.

[5] CMS Collaboration, The CMS experiment at the CERN LHC, JINST 3, S08004 (2008).

[6] T. Sjöstrand, S.Mrenna, and P.Skands, PYTHIA 6.4 Physics and Manual, JHEP05, 026 (2006).

[7] T. Csörgő, S. Hegyi, W.A. Zajc, Eur. Phys. J. C 36, 68 (2004). 